\title{
Diabetes associada à doença periodontal
}

\author{
Diabetes associated with periodontal disease
}

Recebido: 20/10/2021 | Revisado: 25/10/2021 | Aceito: 26/10/2021 | Publicado: 31/10/2021

\author{
Pedro Henrique Barbosa Marine \\ Universidade Brasil, Brasil \\ E-mail: pedrao1999@gmail.com \\ Gabriel Machado \\ Universidade Brasil, Brasil \\ E-mail: machado.gabriel123@hotmail.com \\ Gilson Dantas Junior \\ Universidade Brasil, Brasil \\ E-mail: Juniordantaso804@gmail.com \\ Guilherme Marine \\ Universidade Brasil, Brasil \\ E-mail: Guimarine2209@gmail.com \\ Adalberto Fidalgo da Costa \\ Universidade Brasil, Brasil \\ E-mail: dr.afcosta@terra.com.br
}

\begin{abstract}
Resumo
O presente trabalho tem o objetivo revisar a literatura pertinente no que concernem as possíveis associações entre diabetes e a doença periodontal. O Diabetes Mellitus é uma doença crônica que está afetando a população de forma crescente, tornando-se um sério problema de Saúde Pública. É uma desordem patológica de origem endócrina que provoca inúmeras alterações de ordem sistêmica. Considera-se que o diabetes influencia na instalação e progressão da doença periodontal, a exemplo da dificuldade cicatricial, mas também sofre influência da mesma, posto que o curso clínico da doença periodontal, pode alterar o metabolismo da glicose e consequentemente dificultarem o controle do diabetes. A doença periodontal é uma inflamação resultante da interação entre o acúmulo do biofilme dental e metabólitos bacterianos produzidos sobre a margem gengival, e é mediada pela resposta imunológica do hospedeiro. A ativação do sistema imunológico do hospedeiro, principalmente para fins de proteção, em última instância, resulta na destruição dos tecidos, desencadeando a síntese e a liberação de citocinas, mediadores pró-inflamatórios e as metaloproteinases de matriz. A destruição periodontal vista na periodontite depende do equilíbrio entre a virulência do biofilme local e a resposta imunológica do hospedeiro. A periodontite é considerada um dos principais problemas de saúde em pacientes com diabetes e sua presença pode induzir resistência à insulina. A doença periodontal tem sido apontada como um fator de risco para o controle glicêmico em pacientes descompensados. Desta forma, a estreita relação entre a doença periodontal e diabetes tem sido motivo de preocupação entre os cirurgiões dentistas.
\end{abstract}

Palavras-chave: Doença periodontal; Diabetes; Placa bacteriana.

\begin{abstract}
The present work has the objective to review the relevant literature regarding the possible associations between diabetes and periodontal disease. Diabetes Mellitus is a chronic disease that is increasingly affecting the population, becoming a serious public health problem. It is a pathological disorder of endocrine origin that causes numerous changes of systemic order. Diabetes is considered to influence the onset and progression of periodontal disease, such as scarring, but it is also influenced by it, since the clinical course of periodontal disease may alter glucose metabolism and consequently make it difficult to control diabetes. Periodontal disease is an inflammation resulting from the interaction between the accumulation of dental biofilm and bacterial metabolites produced on the gingival margin, and is mediated by the host's immune response. Activation of the host's immune system, primarily for protection purposes, ultimately results in tissue destruction, triggering the synthesis and release of cytokines, proinflammatory mediators and matrix metalloproteinases. The periodontal destruction seen in periodontitis depends on the balance between the virulence of the local biofilm and the host's immune response. Periodontitis is considered a major health problem in patients with diabetes and its presence may induce insulin resistance. Periodontal disease has been identified as a risk factor for glycemic control in decompensated patients. In this way, the close relationship between periodontal disease and diabetes has been cause for concern among dentists.
\end{abstract}

Keywords: Periodontal disease; Diabetes; Bacterial plaque. 


\section{Introdução}

Dentre as idades de 25 a 44 anos, 17 em cada 1.000 pessoas têm diabetes e 79 em cada 1.00 pessoas com mais de 65 anos tem diabetes. Portanto, cerca de 3\% a 4\% dos pacientes adultos em tratamento odontológico têm diabetes. (SONIS; FAZIO \& FANG, 1966). Incluindo um grupo de distúrbios metabólicos com fenótipo de hiperglicemia (aumento excessivo da concentração de glicose no sangue). Essas doenças podem incluir diminuição da secreção de insulina, diminuição da utilização de glicose e aumento da produção de glicose. (HARRISON et al.,2002).

Pacientes com diabetes apresentam muitas alterações fisiológicas que reduzem sua capacidade imunológica e resposta inflamatória, e aumentam sua suscetibilidade à infecção. (BANDEIRA et al., 2003; CASTILHO \& RESENDE, 1999).

Dente as possíveis doenças sistêmicas nesses pacientes, muitas são as alterações bucais. O objetivo desta revisão é esclarecer a principal correlação entre o diabetes e essas manifestações, e enfatizar as ações que o dentista deve realizar nessa situação.

Além das complicações crônicas, o diabetes mellitus também esta relacionado a complicações bucais. A doença periodontal é a complicação oral mais importante, sendo considerada a sexta complicação clássica do diabetes. A estreita relação entrea instalação e a progressão da doença periodontal e a presença do diabetes mellituscomo fator de colaboração tem sido motivo de preocupação dos cirurgiões dentistas (ALVES, ANDION, BRANDÃO \& MENESES 2006).

O presente trabalho tem como objetivo revisar a literatura e avaliar a doença periodontal associada ao Diabetes Mellitus e suas relações com a cavidade oral.

\section{Metodologia}

Neste estudo foi utilizada como método a análise descritiva a partir de uma pesquisa bibliográfica baseada narevisão de títulos de livros, artigos científicos publicados, teses e revistas especializadas. (PEREIRA et al. 2018).

Para critério de inclusão foi utilizado os seguintes unitermos: Diabetes Mellitus; Periodontia; Placa Bacteriana. Disponível em Medline, Lilacs, Pubmed, Cocrhane, Scielo e BBO, sendo consideradas publicações em inglês, português e espanhol. Os critérios de exclusão foram relatórios e publicações sem fontes confiáveis, materiais com disponibilidade incompleta que não abordava com clareza o tema. Foi analisado um total de 34 publicações.

\section{Revisão de Literatura}

\subsection{Diabetes Mellitus}

Os primeiros registros escritos sobre o diabetes foram encontrados num papiro egípcio datado de 1.500 a. C. Por volta do ano 100 da nossa era, médicos gregos deram o nome “diabetes" à doença. A palavra diabetes significa "sifão”, pois o sinal mais obvia da doença é o aumento no volume da urina. Os médicos gregos observaram também que as formigas eram especialmente atraídas pela urina dos diabéticos. Por volta de 1650, o medico britânico Thomas Willis descobriu o porquê dessa atração das formigas, ao testar a urina de um de seus pacientes diabéticos.

Ele anotou em seus registros que ela era "maravilhosamente doce”. O nome dadoença passou então a ser denominada Diabetes Mellitus, ou seja, "sifão de mel” (MESSINA \& SETCHELL, 2002).

Barcello al. (2000) relataram que muitos pacientes podem apresentar diagnostico de Diabetes Mellitus e não apresentar o quadro clínico tradicional, principalmente àqueles com alterações discretas do metabolismo. Segundo GROSS, em algumas circunstâncias o diagnostico do tipo de Diabetes Mellitus torna-se mais difícil, podendo ser necessária a utilização de alguns exames laboratoriais para estabelecer a possível causa do diabetes, como por exemplo, marcadores de auto- impunidade, medida de auto-anticorpos relacionados à insulite pancreática e a avaliação da reserva pancreática de 
insulina através da medida do peptídeo C e da fase rápida de secreção de insulina. A prevalência de Diabetes Mellitus na populaçãourbana brasileira é de 7,6\% e calcula-se que, em 2025, possam existir cerca de um milhão de diabéticos no país. São mais comuns em afro americanos, hispânicos, nativos norte-americanos, ásio-americanos e originários das ilhas do Pacifico (PERNO, 2001).

O diabetes é uma síndrome heterogênea que resulta de defeitos na secreção ena ação da insulina (REIS et al., 2002 apud FRANCO et al., 2003). A causa da Diabetes Mellitus é desconhecida ou idiopática na maioria dos casos. Porém nesta patologia vários fatores podem estar associados a sua etiologia como o sedentarismo,o estresse, o tabagismo, a idade, a história familiar, o peso e os fatores dietéticos.

É uma doença crônica caracterizada por glicemia consequente a anormalidade no metabolismo de carboidrato, proteína e gordura. As pessoas com diabetes têm organismos que não produzem ou respondem à insulina, um hormônio produzido pelas células beta do pâncreas que é necessário para o uso ou armazenamento de combustíveis corpóreos. Sem a insulina eficiente, a hiperglicemia ocorre e pode levaràs complicações a curto e longo prazo, como a disfunção e falência de vários órgãos (especialmente rins, olhos, nervos, coração e 14 vasos sanguíneos), neuropatia periférica, ulcerações crônicas nos pés, infecções cutâneas de repetição dentre outros. (MAHAN \& ESCOTT-STUMP, 2003).

Para Bjeland et al. (2003), o Diabetes é uma doença crônica e caracterizada pela hiperglicemia e elevação de hemoglobina glicosada. É definido como a insuficiência absoluta ou relativa de insulina, causada por um distúrbio endócrino caracterizado pelas alterações metabólicas dos carboidratos, lipídios e proteínas. A insuficiência de insulina pode ser provocada pela baixa produção pelo pâncreas ou pela falta de resposta dos tecidos periféricos à insulina. Para Moore, Zgibor e Dasanavake (2003), o Diabetes Mellitus caracteriza-se por uma hiperglicemia crônica, onde ocorre uma diminuição da produção de insulina ou pela resistência dostecidos por esse hormônio e pode ser considerado como multifatorial.

O diabetes não é uma doença homogênea e varias síndromes distintas têm sidodelineadas. O National Diabetes Data Group (apud Batista et al, 2005), classifica o diabetes em:

a) Diabetes Mellitus Insulino - dependente (tipo 1): o paciente pode ser de qualquer idade, embora a grande maioria dos casos se desenvolva antes dos trinta anos. São indivíduos geralmente magros e o inicio dos sintomas é usualmente súbito com significativa perda de peso, poliúria e polidipsia. São insulinopênicos com tendência a cetoacidose, portanto, dependentes de terapia com insulina. Apresentam associação com antígeno de histocompatibilidade (HLADW3,DW4), fatores ambientais e genéticos.

b) Diabetes Mellitus (tipo II) (Obeso e não Obeso): manifestam-se geralmente após os trinta anos de idade. Os pacientes podem ser assintomáticos ou levemente sintomáticos, têm frequentemente historia familiar de diabetes e $60 \%$ são obesos. Não tem tendência a cetoacidose, exceto durante períodos de estresse. Não são absolutamente dependentes de insulina exógena para a sobrevivência, embora a terapia com insulina possa ser usada para controlar ahiperglicemia.

Diabetes é considerada um problema de saúde pública por sua magnitude, risco e dificuldades no seu controle. Destaca-se também por sua alta frequência na população, suas complicações, mortalidade, altos custos financeiros e sociais envolvidos no tratamento e deterioração significativa da qualidade de vida (PERES, 2006).

Devido ao aumento de sua incidência, o Diabetes Mellitus é considerado um grave problema de saúde publica. Por ser uma doença sistêmica, tem influencia em todo o organismo, inclusive na cavidade bucal, sendo os pacientes mal controlados, os que têm maior predisposição a apresentarem problemas bucais. Portanto a chance de um cirurgião-dentista se deparar com um paciente diabético acometido por uma avulsão dentaria é cada vez maior, tornando imprescindível o 
conhecimento da enfermidade, de seu tratamento médico e as implicações que ambos podem acarretar no tratamento odontológico. Inclusive faz-se necessário também que o cirurgião-dentista façaparte da equipe multidisciplinar que cuida dos pacientes com Diabetes Mellitus, cabendo ao mesmo, conhecer melhor essa patologia e suas manifestações orais e, estar preparado para atuar em casos de hipoglicemia durante o tratamento, para que possa providenciar cuidados preventivos e terapêuticos mais efetivos. (ALVES et al., 2006).

\subsection{Diagnóstico}

Recentemente, após extensa revisão de evidências de pesquisa do ponto de vista epidemiológico, recomenda - se a utilização do teste de hemoglobina glicosilada (HbA1c) para diagnosticar diabetes com limiar de 6,5\%. (INTERNATIONAL EXPERT COMMITTEE, 2009).

A partir da constatação da glicemia de jejum maios ou igual a $126 \mathrm{mg} / \mathrm{dl}$, confirmada após duas horas com a glicose plasmática maior ou igual 12 a 200mg/dl, é realizado o diagnostico do diabetes (AMERICAN DIABETES ASSOCIATION, 2011a; AMERICAN DIABETES ASSOCIATION,2011b).

\subsection{Diabetes e Doença Periodontal}

\subsubsection{Consequência da diabetes na condição periodontal}

Lalla et. al. (2006) um estudo de caso controle foi feito com o objetivo de analisar a condição bucal de crianças e adolescentes com diabetes. Foram analisados cerca de 182 pacientes com diabetes e 160 controles. Nos grupos a idade dos participantes estava entre 6 e 18 anos de idade. Os resultados mostram que a inflamação gengival das crianças com diabetes foi significamente maior do que o grupo de controle (índice gengival 1.2 x 1.0 e percentual de locais com sangramento de $23.6 \mathrm{x}$ 10.2 para casos e controles, respectivamente). Em comparação com pacientes não diabéticos, crianças e adolescentes diabéticos também apresentaram perda de inserção e número dentes significativamente maiores $(1.8 \times 0.8$ e 5.8 x 1.5 , respectivamente). Após o ajuste para idade, sexo, raça, sangramento gengival e frequência de consultas odontológicas, o diabetes e periodontite ainda estão altamente correlacionados, especialmente no subgrupo de 12 e 18 anos. No grupo de casos,o índice de massa corporal foi significamente relacionado a destruição dos tecidos de inserção, conjuntivo ósseo, mas não ao curso do diabetes e hemoglobina glicosilada média. Os resultados deste estudo mostram que crianças e adolescentes com diabetes apresentam maior dano periodontal. A patogênese do diabetes e da periodontite é bastante complexa.Há evidencias que sugere que os mecanismos do desenvolvimento de outras complicações do diabetes podem estar operando na patogênese de uma periodontite mais acelerada na presença do diabetes.

\subsubsection{Consequência da Condição Periodontal no Controle dos Diabéticos}

A influência do tratamento periodontal no controle do açúcar no sangue frequentemente se reflete nas mudanças nos parâmetros clínicos da inflamação periodontal. Em um estudo de paciente diabético tipo II bem controlados, com gengivite ou periodontite moderada, o tratamento periodontal foi feito com raspagem e alisamento sub-gengival, sem antibióticos. O grupo controle de pacientes diabéticos com condição periodontal semelhante ao que não recebeu tratamento, após três meses a terapia, os pacientes tratados tiveram uma diminuição de $50 \%$ no sangramento gengival. Os mesmos pacientes tiveram uma diminuição significativa na hemoglobina glicada de $7.3 \%$ para 6,5\%. (KIRAN et al., 2005).

Demmer et al., 2008, em um estudo longitudinal, acompanharam por quase duas décadas uma amostra inicial de 9.296 indivíduos. Foi observada uma incidência de $9 \%$ de diabetes no período de acompanhamento de 10 anos. Pacientes que apresentavam periodontite tiveram um aumento de duas vezes mais chances de desenvolverem diabetes. Segundo os autores, 
esta observação é clinicamente relevante, uma vez que é comparável aos riscos associados a outros fatores de risco para o diabetes. Foi demonstrado neste estudo, que os participantes que apresentavam doença periodontal avançada também apresentavam maiores fatores de rico pra diabetes tipo II. Os autorescolocam, ainda que a doença periodontal observada no início do estudo é clinicamente relevante e um novo preditor do diabetes tipo II em uma amostra representativa da população adulta dos Estados Unidos.

Mealey et al. (2006) explica que apenas recentemente começou a se estabelecer mecanismos pelos quais a doença periodontal influi no diabetes. Oprincipal mecanismo referido foi o aumento da resistência tecidual a insulina, levando a um controle glicêmico sanguíneo ruim. Isto possivelmente deve estar relacionado com o envolvimento de citocinas próinflamatórias desencadeadas durante a inflamação, como por exemplo, o TNF- $\alpha$, principalmente no que diz respeito ao diabetes tipo II [29]. Embora alguns estudos tenham apontado quea severidade da doença periodontal esteja relacionada direta ou indiretamentecom o controle glicêmico, ainda não está claro se uma melhora na saúde periodontal seria capaz de aumentar significativamente o controle glicêmico.

\subsubsection{Eficácia da Terapia Periodontal em Indivíduos Diabéticos}

Em uma reanálise de 10 estudos de intervenção, incluindo 456 indivíduos com diabetes tipo I e II, Janket et al., (2005) verificou que, a partir de raspagem e alisamento periodontal, uma diminuição não-significativa de $0,38 \%$ nos níveis de hemoglobina glicada para todos os estudos; $0,66 \%$ nos cinco estudos conduzidos com pacientes somente com diabetes tipo II e $0,71 \%$ quando do uso de antibióticos.

Os autores firmaram que os procedimentos periodontais não afetam o controle glicêmico dos individuos.

Entretanto, Darré et al. (2008) realizaram uma outra metanálise de estudos de intervenção, sobre o efeito da terapia periodontal no controle glicêmico de pacientes diabéticos. Um total de 9 ensaios clínicos com 485 pacientes foram incluídos neste estudo. Os resultados sugerem que a terapia periodontal pode levar a uma redução significativa de $0,79 \%$ nos níveis de hemoglobina glicada.

Recentemente, Teeuw et. al. (2010), publicaram uma meta-análise que visa avaliar como o tratamento periodontal afeta a saúde geral de pacientes diabéticos com melhora do controle glicêmico em um estudo com período de acompanhamento de 3 a 9 meses. Cinco estudos completaram um total de 199 pacientes com periodontite diabética que receberam tratamento periodontal e 183 pacientes com periodontite que não receberam nenhum tipo de tratamento periodontal. Os resultados da meta-análise mostraram que o tratamento periodonta é benéfico para os diabéticos. Além disso, em comparação com os pacinetes que não receberam tratamento, os pacientes com periodontite que receberam tiveram uma redução média de $0,40 \%$ nos níveis de hemoglobina glicosilada. Segundo osautores, essa redução de hemoglobina também é clinicamente significativa, pois qualquer redução levará à redução das complicações do diabetes.

Recentemente, em um ensaio clinico, Koromantzos et.al., (2011) avaliaram o efeito do tratamento periodontal não cirúrgico no níveil dehemoglobina glicosilada em paciente diabético tipo II, com periodontite moderadaa grave. Incluindo 60 pacientes, com idade entre 40 e 75 anos, com níveis de hemoglobina glicosilada entre 7 a $10 \%$ e, pelo menos 16 dentes, no exame periodontal, 8 desses dentes apresentam profundidade de sondagem maior ou igual a 6 mm e perda de inserção clinica maior ou igual a $5 \mathrm{~mm}$, distribuídos em dois quadrantes.

Os pacientes foram divididos aleatoriamente em dois grupos diferentes. Grupo intervenção e grupo controle, e foram acompanhados por 6 meses, com visitas intermediarias no primeiro e terceiro mês. Um único examinador calibrado e cego para os diferentes grupos realizou os exames avaliando profundidade desondagem, perda de inserção clinica, sangramento à sondagem, índice gengival simplificado e numero de dentes ausentes nos quatro exames de cada um dos pacientes. Neste 
estudo, todos os pacientes receberam instrução de higiene bucal no inicio do estudo. O grupo intervenção recebeu tratamento periodontal não cirúrgico na forma de raspagem e alisamento radicular em duassessões, com intervalo de uma semana, através da utilização de ultrassom e instrumentos manuais.

Mais recentemente, Simpson et. al. (2011) realizaram uma meta-análisecom o objetivo de investigar a relação entre terapia periodontal e controle glicêmico em pacientes diabéticos, e identificar a apropriada estratégia de abordagem para esta questão. Foram selecionados ensaios clínicos randomizados de pacientes com diabetes tipo I ou tipo II e com diagnostico deperiodontite. A modalidade de tratamento incluída foi terapia periodontal com debridamento mecânico. Com base nos resultados, os autores concluíram que existe evidencia de melhora do controle metabólico do diabetes após o tratamento da doença periodontal. Os autores ainda propõem que indivíduos com diabetes devem ser aconselhados sobre este aspecto e avaliações a cercada condição bucal devem ser recomendadas como rotina.

\section{Discussão}

Atualmente, se aceita que a Doença Periodontal, é prevalente e mais severa em indivíduos com DM do que nos que não são diabéticos (LOE H., 1993). Tendo em vista a alta prevalência de periodontite em diabéticos, LOE já tinha afirmado que a doença periodontal poderia ser tida a $6^{\mathrm{a}}$ complicação da diabetes.

Phabhu et.al. (1996) afiançaram que os níveis de TNF $\alpha$ e IL-1 $\beta$ são elevados o suficiente para serem "jogados” sistemicamente. As afecções sistêmicas aumentam a resistência do tecido à insulina. O que impede a glicose de adentrar nas células-alvo e trás consigo elevação dos níveis de glicose no sangue, solicitando a produção pancreática aumentada de insulina para manter a glicemia na normalidade.

Em modelo proposto por Grossi e Genco (1998), os seres periopatogênicos podem estimular o ciclo síntese e a secreção das citocinas, e esse ciclo se mostra exacerbado no diabético.

Vassiliadis et. al. (1999) relataram que IK-1 $\beta$ facilita a proteína C quinase de ser ativada e esta destrói células $\beta$ pancreáticas por meio de apoptose celular. Entre os índices em análise, tais marcadores inflamatórios mostraram seus níveis baixos após tratamento periodontal em pacientes diabéticos.

Em sujeitos com periodontite, o estímulo sistêmico persistente de bactérias e seus produtos agem de modo semelhante à infecção sistêmica muito conhecida (IWAMOTO,2001).

Alguns autores resguardam que o efeito do tratamento periodontal no controle do metabolismo do diabete, depende da mortalidade de tratamento escolhido (GROSSI G, 2001).

Entre as terapias, vários pesquisadores designaram a positividade no controle glicêmico à administração sistêmica do antibiótico após terapia periodontal (GROSSI SG, 2001).

Considera-se que a associação tem efeito duplo, diminuindo os pério patógenos no fluido gengival e atuando como modulador da resposta imune dos diabéticos, de forma a inibir a glicação da hemoglobina (GROSSI SG, 2001).

As possíveis trajetórias fisiopatológicas em comum entre Doença Periodontal e Diabetes Mellitus tem sido submetidas a testes por muitos autores, incluindo as associadas à inflamação, a resposta do hospedeiro alterada e à resistência a insulina (ANDERSEN CC, 2006; FLYVBJERG A; 2007; COLE CM, 2008).

A doença DM induz alterações no fenótipo das células imunes e elevação nos níveis séricos de citocinaspróinflamatório. A Periodontite também produz essas problemáticas, no caso de doença periodontal avançada. Muitas moléculas têm sido responsáveis por induzir essa resistência a insulina, como, por exemplo, TNF $\alpha$, IL-6, IL-1 $\beta$ e proteína C reativa (NISHIMURA F, 2007). Entretanto foram encontrados trabalhos em que somente com terapia mecânica tiveram reduções nos níveis de HbA1c nos grupos tratados, quando comparados aos não tratados (KIRAN M, 2005; MAKIURA N, 2008; 
NAVARRO- SANCHEZ AB, 2007).

Em contra mão, alguns investigadores não foram capazes de estabelecer relação entre DP e DM; os valores referentes ao controle de metabolismo permaneceram inalterados após tratamento periodontal (CAMPUS G, 2007; CRUZ GA, 2008). Os níveis microbiológicos demonstraram redução após a terapia periodontal, e não tiveram diferenças entre os grupos diabéticos e não diabéticos, exceto em estudos no qual o envolvimento dos clones tipo II da espécie Porphyromasgengivalis foi relacionado à deteriorização do controle glicêmico (MAKIURA N, 2008).

\section{Conclusão}

A etiopatogênese do diabetes mellitus e da doença periodontal sugeremmecanismos de desenvolvimento semelhantes, o que implica que uma doençapossa estar operando na patogênese da outra, e vice-versa.

Assim, indivíduos com diabetes apresentam maior severidade de doençaperiodontal quando comparados a indivíduos não diabéticos. Da mesma forma, a prevalência da doença periodontal é mais alta nos diabéticos.

O conhecimento da etiopatogênia e progressão da Doença Periodontal,especificamente em relação aos eventos imunes patológicos e inflamatórios, tornam evidente que é possível a periodontite influenciar no controle metabólicodo diabete.

Todavia faz-se imprescindível padronizar as pesquisas no tocante à terapia periodontal e ao grupo examinado (tipo e controle do diabete), com amostras bem maiores para que se consolide a relação bidirecional entre Doença Periodontal e Diabetes Mellitus. A associação epidemiológica entre ambas remete à necessidade do tratamento periodontal no paciente diabético e relevância de enfatizar perante a classe odontológica e medica a importânciade conhecer tal associação, a fim de determinar um plano de tratamento adequado para cada caso.

É de extrema importância que os cirurgiões-dentistas se aprofundem mais no estudo sobre diabetes associada à doença periodontal. Desta forma também se faz fundamental a utilização da técnica empregada com precisão, além da colaboração do paciente, tornando assim um estudo discutido pela comunidade cientifica.

\section{Referências}

ALVES, C., ANDION J., BRANDÃO, M. \& MENESES. Atendimento odontológico do paciente com diabetes mellito: recomendações para a prática clínica. Rev Cienc MedBiol, Salvador, v. 5, n. 2, p.97-110, mai./ago. 2006.

BANDEIRA, F., CALDAS G., FREESEE, GRIZL, FARIA M. \& BANDEIRA C. Endocrinologiane diabetes. Rio de Janeiro: Medsi, 2003. 1109p.

BARCELLOS, I. F.1. Conduta odontológica em paciente diabético. Rev Bras Odontol, Rio de Janeiro, v. 57, n. 6, p. 407-410, nov./dez. 2000.

BATISTA, M.C.R. Avaliação dos resultados da atenção multiprofissional sobre o controle glicêmico, perfil lipídico e estado nutricional de diabéticos atendidos em nívelprimário. Rev. Nutrição, Campinas, v.18, n. 2, p. 219-228, mar./abr. 2005.

BJELLAND, S. Dentists, diabetes and periodontitis. Aust Dent J, Sydney, v. 47, n. 31, p. 202-207, sept. 2003.

CAMPUS G., SALEM A., SACCO G., MAIDA C., CAGETTI M.G. \& TONOLO G. Clinical effects of mechanical periodontal therapy in type 2 diabetic patients. Diabetes Res ClinPract. 2007;75(3):368-9.

DARRÉ L., VERGNES J.N., GOURDY P., \& SIXOU M. Efficacy of periodontal treatment on glycaemic control in diabetic patients: A meta-analysis of interventional studies. Diabetes Metab. 2008; 34(5):497-506.

GROSSI, S.G. \& GENCO R.J. Periodontal disease and diabetes mellitus: a two way relationship. Annals of Period ontology. 1998 Jul;3(1):51-61.

GROSSI,S.G. Treatment of periodontal disease and control of diabetes: an assessmentof the evidence and need for future research. Ann Periodontol. 2001 Dec; 6:138-45.

HARRISON T.R. Medicina Interna. 17 ed. Rio de Janeiro: AMGH Editora Limitada,2008. Vol I e II.

IWAMOTO Y., NISHIMURA F., NAKAGAWA M., SUGIMOTO H., SHIKATA K., MAKINO JANKET, S.J., WIGHTMAN, A., BAIRD, A.E., VAN DYKE, T.E. \& JONES, J.A. Does periodontal treatment improve glycemic control in diabetic patients? A meta-analysis of intervention studies. $J$ Dent Res, v.84, n.12, p. 1154-9, 2005.

JANSSON L., \& HELLERSTROM C. Relationship between oral health and mortality incardiovascular disease. J Clinical Periodontol 2001; 38 : 762-68. 
KHADER Y.S., DAUOD A.S., EL-QADERI S.S., ALKAFAJEI A. \& BATAYHA W.Q. J Diabetes Complications. 2006 Jan-Feb;20(1):59-68.

KIRAN, M., ARPAK, N., ÜNSAL, E. \& ERDOGAN, M.F. The effect of improved periodontal health on metabolic control I type 2 diabetes mellitus. $J$ Clin Periodontol 2005; 32: 266-272.

KOROMANTZOS, P.A. Controlled trial on the effect of non- surgical periodontal therapy in pacients with type 2. part i: effect on periodontal status and glycaemic control. 38 n.2,p 142-147,2011.

LALLA, E., CHENG, B., LAL, S., TUCKER, S., GREENBERG, E., GOLAND, R. \& LAMSTER, I.B. Periodontal changes in children and adolescents with diabetes: a case-control study. Diabetes Care 2006a; 29: 295-299.

LOE, H., Periodontal disease. The sixth complication of diabetes mellitus. DiabetesCare, v.16, n.1, p.329-34, 1993.

MAHAN, L. K., \& ESCOTT-STUMP, S. Krause: alimentos, nutrição \& dietoterapia. 10 ed.São Paulo: Roca, 2003.

MAKIURA N., OJIMA M., KOU Y., FURUTA N., OKAHASHI N., SHIZUKUISHI S. \& AMANO A. Oral Microbiol Immunol. 2008 Aug;23(4):348-51. doi: 10.1111/j.1399-302X.2007.00426.X.

MEALEY, B.L., OCAMPO, G.L. Diabetes Mellitus and Periodontal Disease.Periodontology 2000; 2007; 44: 127-153.

MESSINA, M., \& SETCHELL, K. Soja e diabetes. (Tradução de José Marcos Mandarino,Vera de Toledo Benassi). Londrina: Embrapa Soja, 2002.

MOORE, P. A., ZGIBOR, J. C. \& DASANAVAKE, A. P. Diabetes: A grow epidemic of allages. J. Am. Dent. Assoc., Chicago, v. 134, p. $115-155$, oct. 2003.

NAVARRO-SANCHEZ AB., FARIA-ALMEIDA R. \& BASCONES-MARTINEZ, A. Effect of non-surgical periodontal therapy on clinical and immunological response and glycemiccontrol in type 2 diabetic patients with moderate periodontitis. J Clin Periodontol. 2007;34:835-43.

NISHIMURA, F., IWAMOTO, Y., MINESHIBA, J., SHIMIZU, A., SOGA, Y. \& MURAYAMA, Y. Periodontal disease and diabetes mellitus: the role of tumor necrosisfactor-alpha in a 2-way relationship. J Periodontol, v.74, n.1, p.97-102, 2007.

PEREIRA A. S. (2018). Metodologia da pesquisa científica.

PÉRES, D. S. Comportamento alimentar em mulheres portadoras de diabetes tipo 2.Revista de Saúde Pública, v.40 n.2 São Paulo abr. 2006.

PERIODONTITIS deteriorates metabolic control in type 2 diabetic goto-kakizaki rats. JPeriodontol. 2006;77:350-6.

PERNO, M. A higienista dental: nosso papel na saúde das mulheres - tratando da clientela feminina. Compendium - Ed. Especial: Mulheres e Odontologia, Newtown, v.22, n. 1, p. 45-54, jan. 2001.

PRABHU A., MICHALOWICZ BS. \& MATHUR A. Detection of local and systemiccytokines in adult periodontitis. J Periodontol. 1996;67:515-22.

SIMPSON, T.C. Treatmant of periodontal direase for glycemic control in people withdiabetes v.12, n.5, 2011.

SONIS, STEPHEN T., ROBERT C., FAZIO \& LESLIE FANG. 2 ed Guanabara koogan.1996.

TEEUW, T, J., GERDES, V. \& LOSS B.G. effect of periodontal treatment on glycemiccontrol of diabetic patients a systematic review and Diabetes car, V 33. N 2, P. 421,2010

VASSILIADIS S., DRAGIOTIS V. \& PROTOPAPADAKIS E. The destructive action of IL- 1 alpha and IL-1 beta in IDDM is a multistage process: evidence and confirmation by apoptotic studies, induction of intermediates and electron microscopy. Mediators Inflam. 1999;8:85-91. 